\title{
Conservative Hemodynamic Surgery in the Management of Primary Varicosities of the Long Saphenous Vein: Emerging Experience in Suez Canal University Hospital
}

\author{
Mohamed M. El Yamany ${ }^{*}$, Shaimaa A. Mubarak, Mamdouh M. Almezaien, \\ and Hatem H. Mohamed
}

Department of Surgery, Vascular and Endovascular Unit, Suez Canal University Hospital, Ismailia, Egypt

\begin{abstract}
Background: Contrary to traditional long saphenous vein (LSV) surgeries, stripping and endovenous laser ablation, using heat coagulation, ambulatory conservative hemodynamic correction of venous insufficiency (CHIVA) is LSV sparing technique. Aim: Based on the lack of studies discussing success and recurrence rate, this study was designed to assess the immediate and oneyear follow-up of this technique, as well as its efficacy and safety. Patients and Methods: Thirtythree patients with primary LSV varicosities were included in this uncontrolled clinical trial. $\mathrm{Pa}$ tients with LSV diameter of $\geq 9.5 \mathrm{~mm}$ or pregnancy were excluded. Results: The mean age of our patients was $33.36 \pm 8.865$ years, and $54.5 \%$ of them were males. According to Hobb's score, 25 patients $(75.7 \%)$ were completely cured, 6 patients $(18.1 \%)$ were improved, while 2 patients $(6.2 \%)$ were considered as failure. Most (87.9\%) of the patients showed absent or no visible recurrence. The rest showed visible recurrence by duplex ultrasound. All patients had significant improvement of their symptoms (no pain, or edema) at the immediate follow-up after operation. No complication including hematomas, ecchymosis, hemorrhage, saphenous nerve neuralgia, phlebitis, infection, deep or LSV thrombosis was detected. Conclusion: CHIVA is a successful and effective option in managing patients with primary long Saphenous varicosities, with minimal recurrence rate, after one-year of follow-up.
\end{abstract}

Keywords: CHIVA, recurrence, varicose veins

\section{Introduction}

Varicose veins (Ws) usually presenting with pain, edema, heaviness, and superficial thrombophlebitis, affects the health condition and the quality of life of the patient ${ }^{(1)}$. There are many treatment options. The traditional Long Saphenous vein (LSV) surgical methods with stripping, and the less invasive method as endovenous laser ablation (EVLA), inducing heat coagulation by their catheter are the most common. These previously mentioned operations were built on the idea of disposal of LSV vein to prevent reflux that leads to recurrence of VVs after intervention. A different type of treatment strategy was introduced as ambulatory conservative hemodynamic correction of venous insufficiency (CHIVA) by Franceschi who developed this 
treatment strategy in $1988^{(2,3)}$. CHIVA strategy spares LSV: autologous and best substitute for arterial revascularization of the coronaries and the lower limbs bypass. Duplex ultrasound identifies the refluxing points on LSV that might be linked to varicosities $^{(4)}$. Based on the lack of studies discussing success and recurrence rate. This study was designed to assess the immediate and one-year follow-up of this technique, as well as its efficacy and safety.

\section{Patients and Methods}

\section{Study setting}

Our uncontrolled clinical trial was realized in Suez Canal University Hospital (SCUH), in Vascular and Endovascular Department, between July 2018 and July 2020. This study was approved by the local ethical committee. A written informed consent was obtained from all participants.

\section{Study Population}

Thirty-three patients were randomly selected from those who presented to our outpatient suffering from primary VVs of LSV according to the following inclusion and exclusion criteria. Inclusion criteria: Primary LSV varicosities of Clinical, Etiological, Anatomical and Pathological (CEAP) classification, clinical class $(2-6)^{(5)}$. Presence of sapheno-femoral reflux $>1$ second with or without incompetence of the long Saphenous trunk. Presence of competent and patent deep venous system. One or more incompetent tributaries of the LSV. Exclusion criteria: patients with LSV diameter $\geq$ 9.5mm (Duplex Ultrasound measurement). Pregnant patients. Patients with superficial thrombophlebitism or those on oral anticoagulants.

\section{Pre-operative assessment}

Detailed history, examination, investigations, and radiological assessment were performed to confirm the diagnosis and to exclude patients with exclusion criteria. All patients were assessed with duplex ultrasound (Philips, Clear-Vue 350), by two expert staff of vascular imaging to identify the following points: The sapheno-femoral exact site, its relation in the groin region and its depth from skin surface. Number of inguinal tributaries drain in the saphenofemoral junction (SFJ). Presence of incompetent inguinal tributary. LSV course under the skin (must be marked). Presence of any perforating vein from superficial or deep vein. And the exact LSV diameter. The technique used in this study was the classical technique of Franceschi (CHIVA founder) ${ }^{(6)}$.

\section{Follow-up examination parameters}

Post-operative management

Following treatment, patients wore class 2 medical compression stockings above the knee for three weeks. Patients were discharged from the hospital the same day of surgery. Patients were reviewed in the outpatient clinic in the next day and 7 days later after operation. The follow-up was at 3,6 , and 12 months to assess the outcome clinically and radiologically.

\section{Outcome measures:}

They included incidence of thrombosis of LSV. Vein diameter at fixed points and comparing it to the preoperative values. Clinical recurrence was evaluated based on Hobb's classification: "cure" (absence of VVs), "improvement" (presence of VVs $<0.5 \mathrm{~cm}$ ), and "failure" (presence of VVs $\geq 0.5 \mathrm{~cm}$, main trunks, or incompetent perforators $)^{(7)}$. Radiological recurrence was measured using ultrasonography as: "absent or nonvisible recurrence" (patient clinically cured) and "visible recurrence" (patient in situation of clinical failure), with or without a simple reflux point ${ }^{(7)}$. Complications of the wound (hematomas, ecchymosis, hemorrhage, phlebitis and infection). DVT and LSV thrombosis. 


\section{Statistical analysis}

Data were analyzed using statistical package for the social sciences, version 20 (IBM Corp., Released 2011, IBMSPSS Statistics for Windows, Version 20.0, Armonk, NY: IBM Corp.). Nonparametric Kruskal-Wallis and Mann-Whitney $U$ tests were used for analysis. Likert score averages were given as mean $\pm S D$. $P$ value less than or equal to 0.05 was set as a criterion for establishing statistical significance.

\section{Results}

Between July 2018 and July 2020, 33 consecutive primary varicose vein patients underwent CHIVA procedure at our vascular unit in (SCUH). They were 18 males (54.5\%) with male to female ratio of 6:5. Their age ranged between 25 to 60 years with mean age of $33.36 \pm 8.865$ years. Most of our patients were at the age group from 25 to 45 years. The following risk factors were observed in the studied group of patients. Thirty patients (90\%) had occupations that require standing for long time more than 6 hours per day as hairdressers, barbers, nurses, cookers and teachers. Twenty patients $(60 \%)$ had family history of VVs, 10 of the female patients $(66 \%)$ had multiple pregnancies. There were other risk factors that may affect the outcome: 7 patients (21\%) were diabetics, 5 patients (15\%) were obese with body mass index $(\mathrm{BMI})>30,5$ patients (15\%) were smokers with smoking index > 20, 2 patients (6\%) had IHD (ischemic heart disease). Some patients had more than risk factor. No patient was hypertensive or IKF (impaired kidney function), as shown in table 1.

\begin{tabular}{|c|c|c|}
\hline \multicolumn{3}{|c|}{$\begin{array}{c}\text { Table 1. Distribution of patients according } \\
\text { to their risk factors }\end{array}$} \\
\hline Risk factor & $n$ & $\%$ \\
\hline- Occupation & 30 & $90 \%$ \\
\hline- Family history & 20 & $60 \%$ \\
\hline- Multiple pregnancies & 10 & $30 \%$ \\
\hline- Diabetes & 7 & $21 \%$ \\
\hline- Obesity & 5 & $15 \%$ \\
\hline- Smoking & 5 & $15 \%$ \\
\hline- Ischemic heart disease & 2 & $6 \%$ \\
\hline- Impaired kidney function & 0 & $0 \%$ \\
\hline- Hypertension & 0 & $0 \%$ \\
\hline
\end{tabular}

Regarding the presenting symptoms, most of our patients (60.7\%) presented with pain and edema on standing ( $\mathrm{C}_{3}$ classification), and $(9 \%)$ patients presented with tortuous dilated elongated veins ( $\mathrm{C} 2$ classification), as shown in figure 1. Twenty-four patients (72.7\%) were shunt type 3 (reflux arises at SFJ and flows through the Saphenous trunk into a tributary that eventually drains into the deep system via a perforator), while type $2 \mathrm{~A}$, type $3+2 \mathrm{~A}$ and type $2 \mathrm{~B}$ were (9.1\%) for each type. The classification of the patients regarding the types of shunt, is shown in figure 2. Pre-operative duplex ultrasound was done to assess the LSV diameter at 3 points upper, middle, and lower thigh and reflux time. The mean vein diameter in 5 patients $(15.1 \%)$ was $5 \pm 0.6$ $\mathrm{mm}$, in 10 patients (30.3\%) was $6 \pm 0.6 \mathrm{~mm}$, in 5 patients $(15.1 \%)$ was $7 \pm 0.6 \mathrm{~mm}$, in $10 \mathrm{pa}$ tients $(30.3 \%)$ was $8 \pm 0.6 \mathrm{~mm}$, and in the last 3 patients $(9.1 \%)$ was $9 \pm 0.6 \mathrm{~mm}$, as shown in figure 3. According to CEAP classification, reflux time and vein diameter, 
most of the patients who had large vein diameter had prolonged reflux time and severe clinical symptoms at time of presentation, as shown in table 2 .

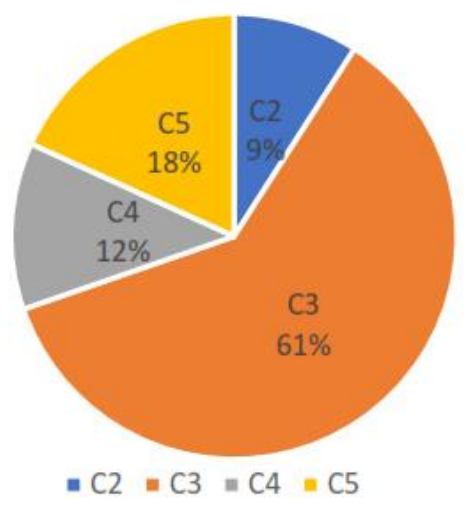

Figure 1: Distribution of patients regarding CEAP classification

Regarding the time of operation, it ranged between 20 and 45 minutes with mean of $30 \pm 5$ minutes. All the patients had spinal anesthesia and they were on supine position. According to the shunt type steps of the operation were determined as the following: 30 patients (90.9\%) who had type 3 shunt, type $2 A+3$ shunt and $2 B$ shunt had ligation and disconnection of SFJ and other refluxing points as the junction was refluxing with preservation of the LSV. Three patients (9.1\%) who had type $2 \mathrm{~A}$ shunt had just ligation of the refluxing points which related to LSV with preservation of SFJ as it was not refluxing. All patients had patent
LSV with no wound complication or anesthesia complication one day after operation. According to Hobb's classification of clinical recurrence rate assessment through, 25 patients (75.7\%) were completely cured, 6 patients (18.1\%) were improved as recurrence was visible vein was $<5 \mathrm{~mm}$ in diameter, and only 2 patients (6.2\%) were considered failure as visible vein was $\geq 5 \mathrm{~mm}$. Regarding recurrence 29 patients (87.9\%) had no visible recurrence, while only 5 patients (12.1\%) showed visible radiological recurrence, documented by duplex ultrasound, as shown in table 3.

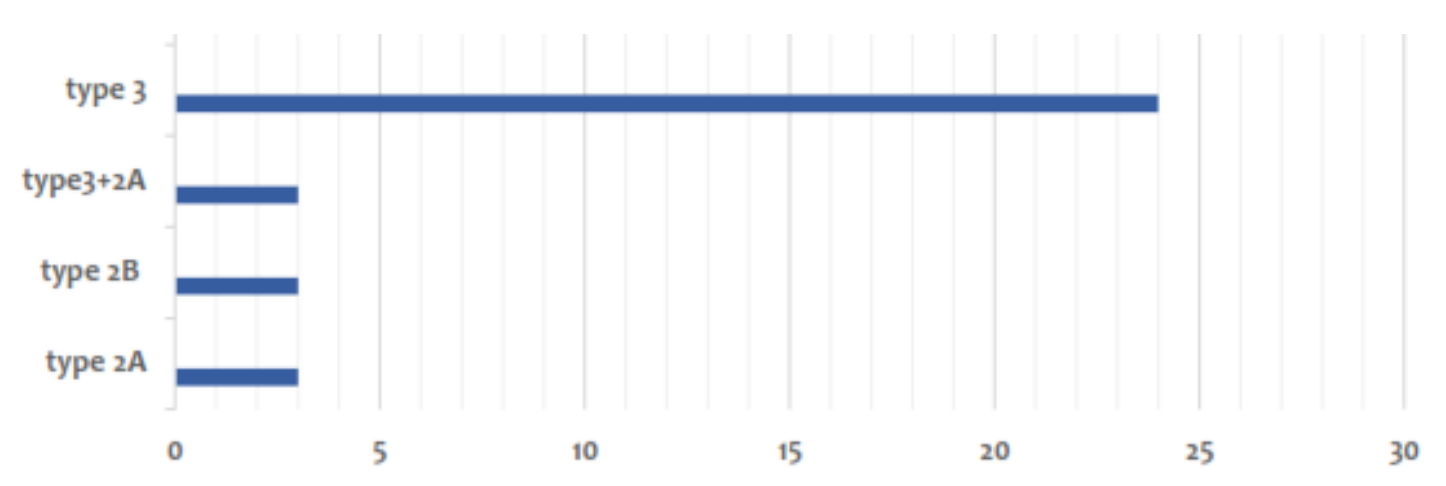

Figure 2: Distribution of patients regarding the types of shunt

Reduction in the vein diameter was the following; patients with mean vein diameter $6 \mathrm{~mm} \pm 0.6$ became $5.5 \mathrm{~mm}$ at the first follow-up after one month, $5 \mathrm{~mm}$ in the second follow-up after 6 months and $4.5 \mathrm{~mm}$ at the last follow-up after one-year. Patients with mean vein diameter $8 \mathrm{~mm} \pm 0.6$ were the same diameter in the 
first follow-up after one-month. It became $7.5 \mathrm{~mm}$ in the second follow-up after 6 months and $7 \mathrm{~mm}$ in the last follow-up after one-year. Patients of mean vein diameter
$9 m m \pm 0.6$ had the same diameter in the first follow-up after one-month. It became $8.5 \mathrm{~mm}$ after 6 months and $8 \mathrm{~mm}$ after oneyear in the last follow-up (figure 4).

\begin{tabular}{|c|c|c|c|c|}
\hline$n$ & Reflux time & Mean vein diameter & Clinical presentation & $\%$ \\
\hline 5 & 2 seconds & $5 \mathrm{~mm}$ & $\mathrm{C}_{2}$ & $15.1 \%$ \\
\hline 5 & 3 seconds & $7 \mathrm{~mm}$ & $C_{3}$ & $15.1 \%$ \\
\hline 10 & 3 seconds & $6 \mathrm{~mm}$ & $C_{3}$ & $30.3 \%$ \\
\hline 13 & (4-5) seconds & $(8-9) \mathrm{mm}$ & $\mathrm{C}_{4}$ and $\mathrm{C}_{5}$ & $39.4 \%$ \\
\hline
\end{tabular}

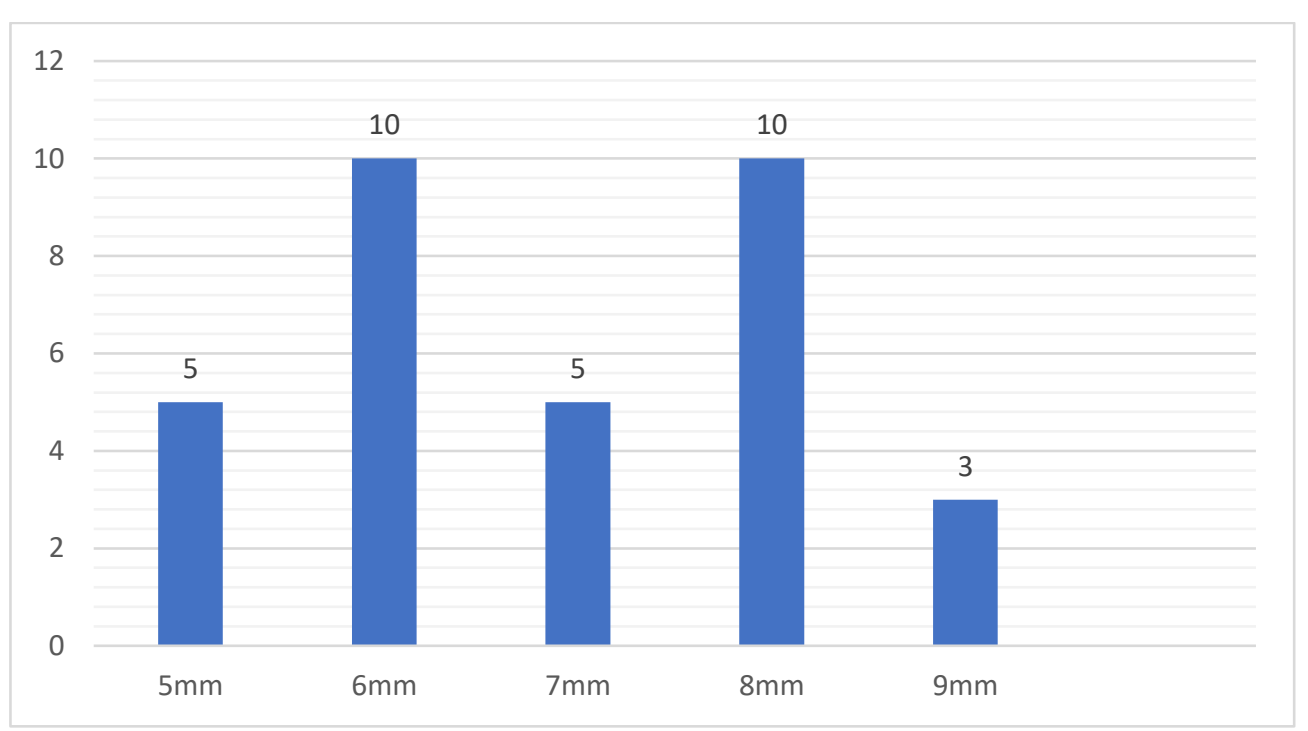

Figure 3: Distribution regarding mean LSV diameter

All patients had no infections, with significant improvement of their clinical presenting symptoms (no pain, or edema) detected by examining the patients in the follow-up visits. No complications including hematomas, ecchymosis, hemorrhage, Saphenous nerve neuralgia, phlebitis, deep venous thrombosis and or thrombosis were detected.

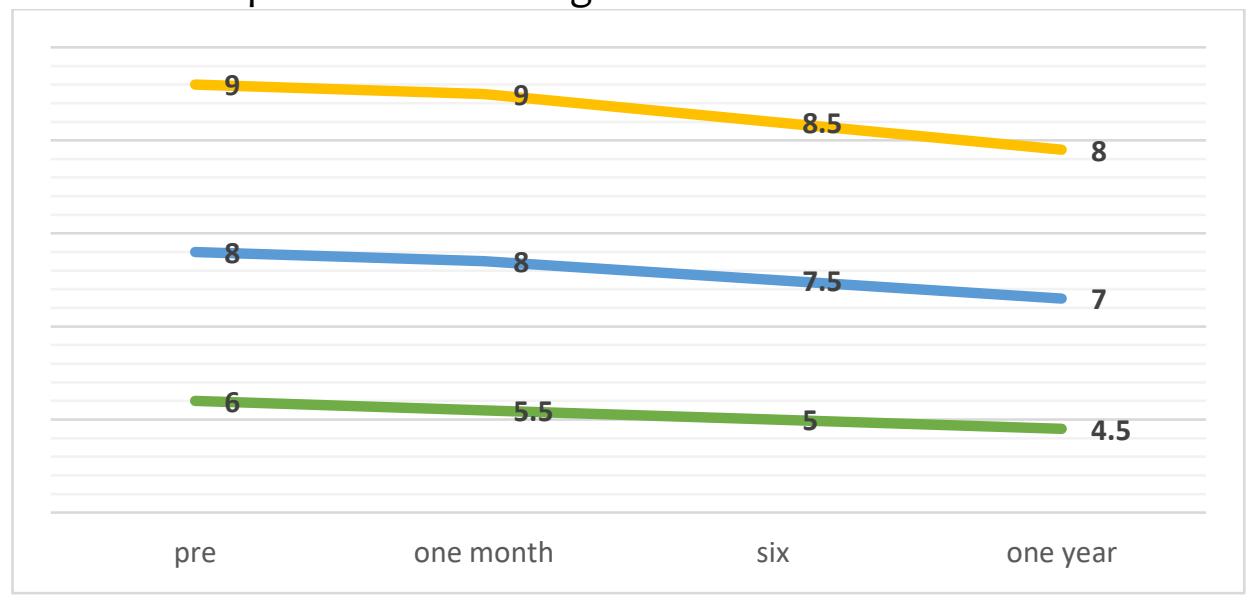

Figure 4: Mean reduction of the vein diameter 


\begin{tabular}{|c|c|c|}
\hline \multicolumn{3}{|c|}{$\begin{array}{c}\text { Table 3. Clinical and radiological recurrence rate } \\
\text { after one-year follow-up }\end{array}$} \\
\hline Recurrence rate & $\mathrm{N}$ & $\%$ \\
\hline$-\quad$ Cure & 25 & $75.7 \%$ \\
\hline- Improved & 6 & $18.1 \%$ \\
\hline- Failure & 2 & $6.2 \%$ \\
\hline Radiological (duplex ultrasound assisted) & & \\
\hline$-\quad$ No visible recurrence & 29 & $87.9 \%$ \\
\hline$-\quad$ Visible recurrence & 5 & $12.1 \%$ \\
\hline${ }^{*}$ Clinical assessment regarding Hobb's classification $(7)$ \\
\hline \multicolumn{3}{|l|}{} \\
\hline
\end{tabular}

\section{Discussion}

Traditional and less invasive surgeries provide LSV stripping or thermal ablation to avoid VVs recurrence. And this condition is a grave to patients with ischemic heart disease or peripheral arterial disease. Sparing LSV is the main idea of CHIVA technique, in the future as a natural graft either in coronary bypass or graft for arterial repair in case of traumatic arterial injury or bypass in case of chronic ischemia( ${ }^{(8)}$. As this technique is new and until now still under research, our study was designed to search for the rate of success of this technique regarding the clinical and radiological recurrence and its safety and efficacy. The demographic data in our study showed that males are more affected than females but without big difference in percent $54.5 \%$, $45.4 \%$ respectively. This may reflect that males in our country more exposed to jobs that need long standing duration for more than 6 hours per day but females also exposed to other risk factor which was multiple pregnancies .That was contradicted with the studies conducted in Spain by Escribano et al. ${ }^{(8)}$, $62 \%$ females, $38 \%$ males and Fernandez et al.(10), 89\% females, $11 \%$ males that females were predominate in VVs without logical explanation. Regarding the mean age, there was another difference between our study ( 33.36 years) and the study done in Germany ( 52.7 years) by
Mendoza. ${ }^{(9)}$ That might be explained more jobs needing long hours of work while standing and for long durations, compared to the other countries. Regarding the presenting symptoms, most of our patients were in $\mathrm{C}_{3}$ category followed by $\mathrm{C}_{4}, \mathrm{C}_{5}$ and leastly C2. This disagreed with the study conducted in Spain by Fernandaz et al. ${ }^{(10)}$, as most of their patients were in $\mathrm{C} 2$ category, followed by $\mathrm{C}_{3}$ and least of them were in category $\mathrm{C}_{4}$ and $\mathrm{C}_{5}$. That might be due to late seek of medical advices in our country: only when they had symptoms that affect their daily normal activities and lor when symptoms were advanced. Another explanation is the economic reasons, as most of these patients did not have any medical insurance. Regarding the type of shunts according to duplex, most of the patients in our study were type 3 shunt, followed by type 2 shunt, a contradiction with the study conducted in china by Hua wang et al. (11), as most of their patients were had type 1 shunt followed by type 3 shunt. This may be due to racial causes. Regarding the vein diameter, most of our patients had vein diameter more than $6 \mathrm{~mm}$. that was contradictory with the study conducted in Brazil( $^{(12)}$, where most of the patients had vein diameter $<5 \mathrm{~mm}$. That might be due to late seek of medical advices in our country, this due to economic reasons, as most of these patients did not have any medical insurance as before. 

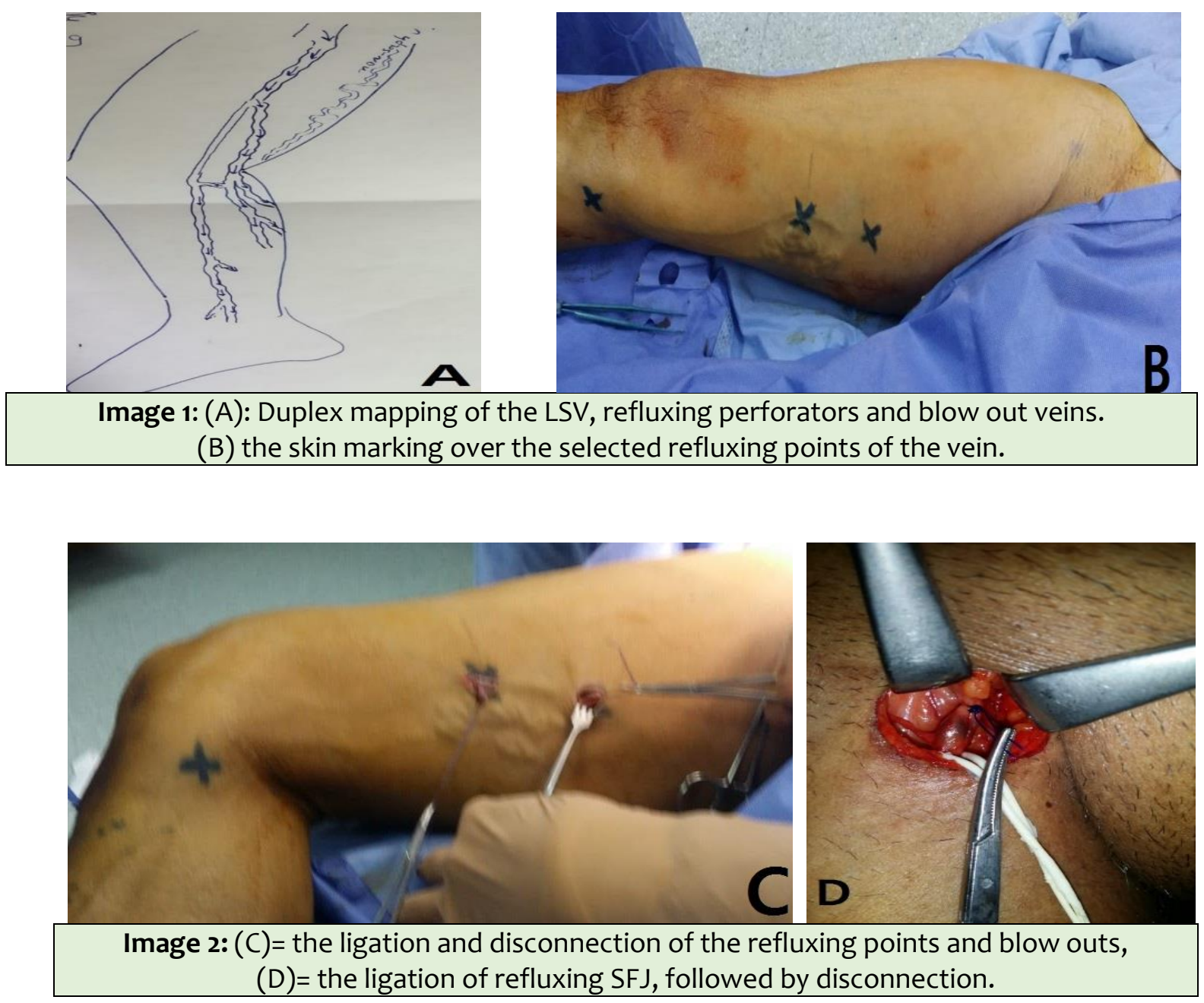

This result in larger mean vein diameter than in other countries and more advanced categories $\mathrm{C}_{3}, \mathrm{C}_{4}$, and $\mathrm{C}_{5}$ as well. In our study, we found that there was a direct relation between clinical presentation of the patient, mean vein diameter, reflux time and mean reduction of vein diameter; most of the patients who had large vein diameter had also prolonged reflux time and severe symptoms during presentation with longer time post-operatively for diameter reduction. This may be due to delayed presentation and weak redundant venous wall from prolonged reflux. Regarding the mean reduction of vein diameter, the reduction was greater in veins $<8 \mathrm{~mm}$ in diameter compared to vein diameter $>8 \mathrm{~mm}$. This may reflect that when the vein diameter is large its wall is redundant and has less elasticity, so after reflux elimination postoperatively the mean reduction is small and vice versa. The mean reduction of vein diameter in our study was $1.5 \mathrm{~mm}$ after oneyear follow-up that disagreed with the study conducted in Spain by Escribano et al. ${ }^{(8)}$, where the mean reduction in the LSV diameter was $2.7 \mathrm{~mm}$ after 3 years followup. This may be due to different follow-up period between both studies, shorter in our study. The other possible cause might be the larger diameter of the vein in our study necessitating longer period. In our study, the absence of wound complications was related to the minimally invasive surgery (CHIVA), that attributed to the small wound size, minimal tissue dissection and the ligation of SFJ without stripping which lead to avoid post-operative early complications as ecchymosis, hematomas, infection and numbness. All patients had follow-up after one-week then 3, 6 and 12 months after operation. There was no late 
complication detected during the followup period (no DVT, numbness or thrombophlebitis). That agreed with the study conducted by Zamboni et al.(13), as there was no late complication detected through their follow-up period which was more than ours.

\section{Conclusion}

Based on our results CHIVA seems to be successful, safe and effective method in managing patients with Ws with extremely rare complication and minimal recurrence rate.

Financial support and sponsorship: None

\section{Conflicts of interest: None}

\section{Acknowledgement}

Authors would like to express their thankfulness and gratitude to Professor Amr MOGHAZY for reviewing as well as linguistic and scientific editing of this work, and Professor Sherif REFAAT for his guidance during the study and his great help in surgeries.

\section{References}

1. Muhlberger D, Morandini L, Brenner E. (2009) 'Venous valves and major superficial tributary veins near the sapheno-femoral junction`. J Vasc Surg;49(6): pp 1562-1569.

2. Carandina S, Mari C, De Palma M. (2008) Varicose Vein Stripping vs Hemodynamic Correction (CHIVA): a long-term randomized trial'. Eur J Vasc Endovasc Surg;(2): pp 230-237.

3. Cappelli M, Molino L, Ermini S. (2000) 'Ambulatory conservative hemodynamic management of varicose veins: critical analysis of results at 3 years`. Ann Vasc Surg;14(4): pp 376384.

4. Salvatore $G$, Milone $M$, Maietta $P$, Fernandez L, Milone F. (2011) 'Recurrent varicose --veins of the lower limbs after surgery: role of surgical technique (stripping vs. CHIVA) and surgeon's experiences `Chir;32(7): pp 460-463.

5. Corcos L, De Anna D, Dini M, Macchi C, Ferrari P, Dini S. (2000) 'Proximal long Saphenous vein valves in primary venous insufficiency. J Mal Vasc;25: pp 27-36.

6. Gianesini $S$, Occhionorelli $S$, Menegatti $E$, Zuolo $M$, Tessari $M$, Spath $P$. (2015) CHIVA strategy in chronic venous disease treatment: instructions for users` phlebology;(3): pp 157-171.

7. Hobbs J. (1974) 'Surgery and sclerotherapy in the treatment of varicose veins'. Arch Surg; (109): pp 793-796.

8. Escribano J, Juan J, Bofill R, Maeso J, Rodriguez-Mori A, Matas $M$. et al. (2003) 'Durability of reflux-elimination by a minimal invasive CHIVA procedure on patients with varicose veins. A 3-year Prospective Case Study`. Eur J Vasc Endovasc Surg; (25): pp 159-163.

9. Mendoza E, Blattler W, Amsler F. (2012) 'Great saphenous vein diameter at the saphenofemoral junction and proximal thigh as parameters of venous disease class`. Euro J. of Vasc Endovasc Surg;45(1): pp 76-83.

10. Fernandez $N$, Linares-Palomino $P$, Lopez-Espada C, Martinezga F, RosDie E. (2016) 'Clinical results of a new Strategy (modified CHIVA) for surgical treatment of anterior accessory great saphenous varicose veins`. Cirugía Española;94(3): pp 144-150.

11. Hua Wang $Y$, Qianyi $C$, Zhewei F, Endong $\mathrm{Z}$, Zhanghui $\mathrm{Y}$, Xiaowang $\mathrm{H}$. (2016), 'Hemodynamic classification and CHIVA treatment of varicose veins in lower extremities', Int J Clin Exp Med;9(2): pp 2465-2471.

12. De Moraes M, Fuji E, Figueiredo F, et al. (2018) immediate and long-term evaluation after surgery on the saphenous vein with the modified CHIVA technique`. Scripta Scientifica Vox Studentium;2: pp 7-12 
13. Zamboni P, Cisno C, Marchetti F, Quaglio D, Mazza P, Liboni A. (2001) 'Reflux elimination without any ablation or disconnection of the saphenous vein. A hemodynamic model for venous surgery. Eur J Vasc Endovasc Surg;21: pp 361-369. 